\title{
Corrigendum: CLARITY for mapping the nervous system
}

\author{
Kwanghun Chung \& Karl Deisseroth \\ Nat. Methods 10, 508-513 (2013); published online 30 May 2013; corrected after print 20 June 2013
}

In the version of this article initially published, several reference callouts in the text were wrong. The errors have been corrected in the HTML and PDF versions of the article.

\section{Corrigendum: Whole-rat conditional gene knockout via genome editing}

Andrew J Brown, Daniel A Fisher, Evguenia Kouranova, Aaron McCoy, Kevin Forbes, Yumei Wu, Rachel Henry, Diana Ji, Andre Chambers, Joe Warren, Weiguo Shu, Edward J Weinstein \& Xiaoxia Cui

Nat. Methods 10, 638-640 (2013); published online 9 June 2013; corrected after print 26 August 2013

In the version of this article initially published, a name was misspelled in the Acknowledgments section. The error has been corrected in the HTML and PDF versions of the article.

\section{Retraction: Marker-independent identification of glioma-initiating cells}

\author{
Virginie Clément, Denis Marino, Cristina Cudalbu, Marie-France Hamou, Vladimir Mlynarik, Nicolas de Tribolet, Pierre-Yves Dietrich, \\ Rolf Gruetter, Monika E Hegi \& Ivan Radovanovic
}

Nat. Methods 7, 224-228 (2010); published online 21 February 2010; retracted 8 September 2013

In the version of this article initially published, we described a method to identify tumor-initiating cells from human primary gliomasphere cell cultures or directly from fresh human glioma specimens. We used FACS analysis to describe a tumor-initiating cell subpopulation that displayed a specific morphology (high forward scatter, low side scatter) and had high fluorescence in the FL1 channel of the FACS (excitation at $488 \mathrm{~nm}$, emission at $520 \mathrm{~nm}$ ). We have since become aware that the majority of the primary gliomasphere lines (7 of 10 ) used in this paper were contaminated with HEK cells expressing GFP. We had used these sphere cultures to illustrate the selection method, to measure self-renewal, to assess the expression of stemness genes and to test for tumorigenicity.

As assessed by microsatellite analysis of 15 short tandem repeats, seven of the primary sphere cultures used in this paper and all of the three lines used for tumorigenicity experiments did not match their parental tissue, and their genetic profile was consistent with that of HEK cells. We further observed expression of GFP mRNA with reverse-transcription PCR in 7 of the 10 gliomasphere lines. Two of these lines (GSM-1 and OA-III-1) were traceable by microsatellite analysis to their parental tissue at low passages (2-9) but not at higher passages ( $>20$ ), a result showing that contamination occurred during passage of the cells in the laboratory.

We also described experiments done with cells prospectively isolated from freshly resected glioma tissues; we believe that these experiments remain valid. We discussed in the paper that these cells were rare and did not have the same high fluorescence as gliomasphere cultures. We speculated that glioma cells acquire high fluorescence under in vitro conditions. This led to our misinterpretation of the progressive increase in fluorescence of two of the gliomasphere cultures that were in fact being progressively overgrown by contaminating GFP-expressing HEK cells.

All together, when we exclude HEK-contaminated cultures, we must conclude that glioma-initiating cells do not have high autofluorescence levels or acquire them during culture. Selection of a tumorigenic fraction may be possible on the basis of morphological characteristics. However, because an important part of the study was done on contaminated cells, we wish to retract the paper. We deeply apologize to the scientific community for erroneously reporting an artifactual phenomenon as well as for the delay in detecting, characterizing and reporting the error.

Cross-contamination of cell cultures is likely to be a frequent problem. Routine tracing of cell lines by microsatellite analysis has been advocated before publishing work with cell lines. Our experience shows that this advice holds true also for primary cells passaged in culture. 